\title{
HseSUMO: Sumoylation site prediction using half-sphere exposures of amino acids residues
}

\author{
Alok Sharma ${ }^{1,2,3^{*+}} \mathbb{D}$, Artem Lysenko ${ }^{2 \dagger}$, Yosvany López ${ }^{4}$, Abdollah Dehzangi ${ }^{5}$, Ronesh Sharma ${ }^{3,6}$, Hamendra Reddy ${ }^{3}$, \\ Abdul Sattar ${ }^{1}$ and Tatsuhiko Tsunoda $a^{2,7,8^{*}}$
}

From 17th International Conference on Bioinformatics (InCoB 2018): Genomics

New Delhi, India. 26-28 September, 2018

\begin{abstract}
Background: Post-translational modifications are viewed as an important mechanism for controlling protein function and are believed to be involved in multiple important diseases. However, their profiling using laboratorybased techniques remain challenging. Therefore, making the development of accurate computational methods to predict post-translational modifications is particularly important for making progress in this area of research.

Results: This work explores the use of four half-sphere exposure-based features for computational prediction of sumoylation sites. Unlike most of the previously proposed approaches, which focused on patterns of amino acid co-occurrence, we were able to demonstrate that protein structural based features could be sufficiently informative to achieve good predictive performance. The evaluation of our method has demonstrated high sensitivity (0.9), accuracy (0.89) and Matthew's correlation coefficient (0.78-0.79). We have compared these results to the recently released pSumo-CD method and were able to demonstrate better performance of our method on the same evaluation dataset.
\end{abstract}

Conclusions: The proposed predictor HseSUMO uses half-sphere exposures of amino acids to predict sumoylation sites. It has shown promising results on a benchmark dataset when compared with the state-of-the-art method. The extracted data of this study can be accessed at https://github.com/YosvanyLopez/HseSUMO.

\section{Background}

Post-translational modifications (PTMs) of proteins are enzyme-medicated covalent alterations of protein sequence during which a chemical group can be added to a particular residue or sequence is cleaved at a specific location [1]. These modifications greatly expand the range of possible final forms of proteins that can be generated from the same genomic sequence [2]. PTMs also play an important role in modulation of all aspects of protein function, in particular, they can determine protein localization within

\footnotetext{
* Correspondence: alok.sharma@griffith.edu.au; tsunoda.mesm@mri.tmd.ac.jp ${ }^{+}$Alok Sharma and Artem Lysenko contributed equally to this work. ${ }^{1}$ Institute for Integrated and Intelligent Systems, Griffith University, Q, Brisbane LD-4111, Australia

${ }^{2}$ Laboratory for Medical Science Mathematics, RIKEN Center for Integrative Medical Sciences, Yokohama, Kanagawa, Japan

Full list of author information is available at the end of the article
}

the cell [3], mediate signal transduction $[4,5]$, activate or deactivate enzymes and transporters $[6,7]$ and underlie protein degradation and recycling [8]. Despite this critical role PTMs play in all living systems, accurate identification of all types of these modifications using laboratory methods remains challenging. Some pertinent problems include [9]: (1) isolation of specific proteins with modification (s) of interest from the highly diverse and biochemically heterogeneous proteome (2) masking effects of the highly abundant proteins in the sample, which can make isolation using standard immunoprecipitation and chromatographic methods difficult and (3) the diversity and molecular complexity of possible PTMs themselves.

Positions at which PTMs are possible are usually highly specific to particular protein residues and are governed by the amino acid motifs at the site and three-dimensional

(c) The Author(s). 2019 Open Access This article is distributed under the terms of the Creative Commons Attribution 4.0 International License (http://creativecommons.org/licenses/by/4.0/), which permits unrestricted use, distribution, and reproduction in any medium, provided you give appropriate credit to the original author(s) and the source, provide a link to the Creative Commons license, and indicate if changes were made. The Creative Commons Public Domain Dedication waiver (http://creativecommons.org/publicdomain/zero/1.0/) applies to the data made available in this article, unless otherwise stated. 
conformation of the protein [1]. Therefore, a priori identification of the sites and proteins where particular PTMs can occur can be an important way to narrow down the possibilities and facilitate their experimental verification. This sequence-based specificity allows for the possibility of identification of these sites using computational predictive approaches and development of such methods is an area of active research [10].

One of the important types of PTMs, called sumoylation, involves reversible covalent bonding of small ubiquitin-like modifier proteins SUMO1, SUMO2 and SUMO3 [11]. This modification is of particular interest due to its involvement in neurodegenerative [12-14] and immune-related diseases [15-17], as well as cancer [18, 19]. Sumoylation involves several enzymatic steps and was previously reported to be commonly found at $\psi$ K.E/D (or inverse E/D.K $\psi$ ) motif, where $\psi$ can any of the hydrophobic amino acids [20]. However most recent results indicate that as many as half of all sumoylation sites do not actually follow this pattern [4], therefore simple motif-based strategies are likely to be insufficient for good predictive performance on these more recent data.

To date, multiple sequence-based approaches to prediction of sumoylation sites have been proposed. Some earlier approaches are SUMOsp [21], which employs a groupbased prediction system (GPS) similarity clustering and SUMOpre [22], which works by fitting a multiple linear regression model to a 7-residue sequence window. In 2009, an updated version of the SUMOsp algorithm was released that achieved higher specificity and accuracy than any other tools available at the time and proved the utility of their original GPS-based approach. Next, SUMOhydro [23] was proposed that introduced an improved dataset of experimentally-determined sumoylation sites and explored an original type of predictive feature (binary-encoded hydrophobicity pattern) in combination with a support vector machine classifier. GPS-SUMO [24] further refined a GPS-based approach proposed in SUMOsp 1.0 and 2.0, and combined it with particle swarm optimization algorithm. Then, an important shift in direction was proposed by [25], who were the first to use protein structural features for prediction of sumoylation sites, in particular, predicted disorder and confirmation flexibility. And, finally, the most recent methods are pSumo-CD [26] and SUMO_LDA [27]. The former applied a covariance discriminant algorithm in combination with pseudo amino acid composition model. The latter works by computing a position-specific amino acid propensity matrix at locations of interest, which was then used in combination with linear discriminant analysis.

Although good performance results have been consistently reported even in the very early studies, it appears likely that these efforts have been severely limited by the amount of experimental data that was available at the time. Most importantly, it appears that the proportion of the sumoylation sites that do not follow the consensus motif was previously greatly underestimated (e.g. it was assumed to be only about $23 \%$ in [21]) until sufficient data were collected in more recent studies [4]. This indicates that the problem is both more complex than was previously thought and that structural patterns of the protein fold likely play an important role in determining suitable sumoylation sites. However, a vast majority of previously proposed methods have focused on features chiefly based on amino acid occurrence patterns at the sites of interest. To explore the usefulness of structural features [28-33], we propose a novel method, HseSUMO that uses a combination of four different half-sphere exposure (HSE) measures, originally developed to characterize solvent exposure at particular amino acid residues [34]. We demonstrate that a combination of these features is highly promising for prediction of sumoylation sites and we were able to achieve very good levels of performance even using a relatively simple decision tree classifier $(0.89$ area under ROC curve for 6,8 and 10-fold cross-validation schemes).

HSE measure is used for feature computation. It is an alternative measure of the solvent exposure of the amino acids and its use for sumoylation site prediction has shown promising results when compared with the existing state-of-the-art sumoylation site predictor. This indicates that HSE contains the complementary information of the amino acids to identity the sumoylation sites. In the literature, the HSE measure has been shown to contain important information in the related field of research $[35,36]$.

In addition, the imbalance of data deteriorates the performance. To tackle this, we have used the under-sampling technique to obtain the balanced sampling. Furthermore, to report the statistical significance of the HseSUMO, we performed k-fold cross-validation and reported performance measures such as sensitivity, specificity, accuracy and MCC. For sumoylation site prediction, it is very crucial to have high sensitivity as detecting affected lysine sites are of prime importance. Thus, HseSUMO achieved performance improvement of $36.5 \%$ in terms of sensitivity and $16.7 \%$ in terms of accuracy.

\section{Methods \\ Dataset description}

All data used in this analysis is from the Compendium of Protein Lysine Modifications (CPLM) database [37], a resource which manually curates information about 12 different types of lysine PTMs from literature. From these data we have identified a subset of all proteins which were profiled for sumoylation, giving 448 proteins in total that had 780 positive examples of sumoylated sites and 21,353 confirmed non-sumoylated sites. We filtered out sequences over $40 \%$ sequential similarity using CD-HIT [38]. Therefore, our benchmark dataset has less 
than $40 \%$ sequential similarity. The difference between positive and negative samples creates class imbalance problem. The two commonly used strategies to overcome this problem are over-sampling and under-sampling. The over-sampling procedure could increase the probability of over-fitting the model, and under-sampling often provides a modest solution for a given model. Therefore, we selected under-sampling procedure like NearMiss method [39] by employing the imbalanced-learn package of python. After applying NearMiss method we ended up with 780 negative and 780 positive samples.

\section{Half-sphere exposure feature computation}

Half-sphere exposure (HSE) is a solvent exposure measurement similar to Contact Number (CN) [40], and Accessible Surface Area (ASA) [41]. In contrast to ASA and CN that do not provide explicit information regarding the orientation of side chains which is important on the conformation of the 3D structure of the proteins and its interaction with other macro-molecules HSE is designed to attain such information [42]. HSE is introduced in [42] and can be calculated in two ways by splitting the sphere around the $C \alpha$ atom (with radius $\mathrm{R}$ typically equal to $12 \AA$ ) into two half-spheres either along the vector of $C \alpha-C \beta$ atoms or a pseudovector of $C \alpha-C \beta$ generated from the sum of vectors $C \alpha_{i-1}-C \alpha_{i}$ and $C \alpha_{i+1}-C \alpha_{i}$. The first one is referred to as HSE $\beta$ and the second one as HSE $\alpha$.

For HSE $\beta$, the half-sphere containing the $C \beta$ atom is then defined as upper and the other as down half-spheres and the numbers of $C \alpha$ atoms enclosed in these two half-spheres were named as HSE $\beta$-up and HSE $\beta$-down, respectively. An illustration for HSE is given in Fig. 1. For HSE $\alpha$, the half-sphere perpendicular to the sum of $C \alpha_{i-1}-C \alpha_{i}$ and $C \alpha_{i+1}-C \alpha_{i}$ vectors is HSE $\alpha$-up and another one is HSE $\alpha$-down. The main difference between HSE $\beta$ and HSE $\alpha$ is that calculating the second does not require the position of $C \beta$ which is hard to determine for some cases. It was shown in [42] that HSE $\alpha$ is a better measurement for solvent exposure than CN, ASA and even HSE $\beta$. However, the use of HSE $\alpha$ and HSE $\beta$ simultaneously has shown to be complementary [43, 44].

\section{Lysine residue description}

Lysine residue (sumoylated or non-sumoylated) is described by a segment of 31 amino acids ( 15 upstream and 15 downstream) as done by previous studies [45-51]. If a lysine residue is present in any terminus of a protein sequence and if the segment of 31 residues is not possible then we adjusted the segment by employing mirror of amino acids [45]. Suppose lysine residue site is denoted by $K$ then a segment of 31 amino acids will be given as $S=\{A$ $\left.{ }_{-15}, A_{-14}, \ldots, A_{-1}, L, A_{1}, \ldots, A_{14}, A_{15}\right\}$. Therefore, a lysine $K$ consists of 31 HSE features and it is characterized by the 124-dimensional feature vector. The high dimensionality can be further reduced by feature selection techniques [52-54].

\section{Model training}

The model was trained using a decision tree-based classifier. Despite its simplicity, the decision tree classifier has the advantage of allowing easy interpretation of the underlying model, which can facilitate discovery of biologically meaningful patterns captured by the model. To get an accurate measure of performance we have used a repeated cross-validation approach. There are three cross-validation methods, i.e., independent dataset test, sub-sampling (or K-fold cross-validation) test, and jackknife test, often used to evaluate the anticipated success rate of a predictor. Among the three methods, however, the jackknife test is deemed the least arbitrary and most objective one and hence has been widely recognized and increasingly adopted

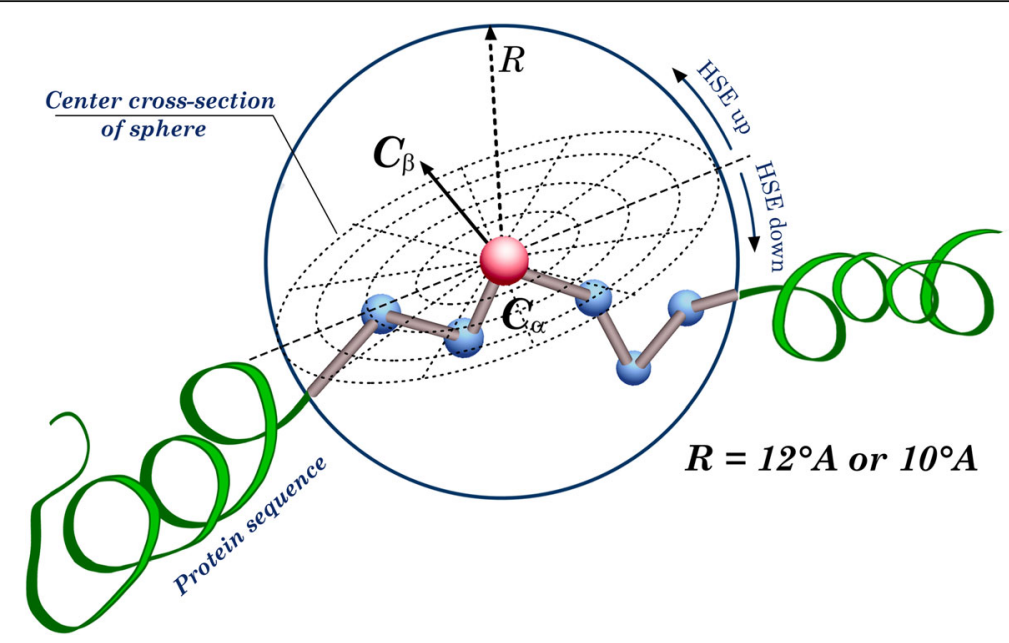

Fig. 1 An illustration of half-sphere exposure of amino acid 
by investigators to examine the quality of various predictors [55-59]. In the jackknife test, each sequence in the training dataset is in turn singled out as an independent test sample and all the rule-parameters are calculated without including the one being identified. Though Jackknife test is the most effective, due to its computational expense we have adopted the K-fold test which is an alternative evaluation method and has been widely used in many protein-related problems [33, 60-62]. Briefly, in K-fold the dataset was partitioned into $k$ approximately equally-sized folds (in this case either 6,8 or 10) [63]. In turn, each of the subsets was set aside and used for validation of the model trained on the remaining $k-1$ folds. To train the predictor, we used the Python implementation of decision trees. The quality of a split was measured with Gini impurity and "best" was selected as the strategy for choosing the split at each node. Moreover, only two samples were required for splitting an internal node.

\section{Evaluation of model performance}

According to established evaluation framework, we define the number of instances where sumoylated sites were predicted, either correctly or incorrectly as true positives (TP) or false positives (FP) respectively. Similarly, for non-sumoylated sites, counts of correct or incorrect predictions are defined as false negatives (FN) or true negatives (TN). Then sensitivity (also known as true positive rate) is a proportion of correctly predicted sumoylated sites among all real sites:

$$
\text { Sens }=\frac{T P}{F N+T P}
$$

and specificity is a proportion of all correctly predicted non-sumoylated sites among all negative predictions:

$$
\text { Spec }=\frac{T N}{F P+T N}
$$

The other two performance metrics are accuracy and Matthew's correlation coefficient which are defined according to the following formulae:

$$
\begin{aligned}
& \text { Accuracy }=\frac{T N+T P}{T N+F N+T P+F P} \\
& M C C=\frac{(T P \times T N)-(F P \times F N)}{\sqrt{(T N+F P) \times(T P+F N) \times(T P+F P) \times(T N+F N)}}
\end{aligned}
$$

The last evaluation metric used was the area under receiver-operator characteristic (ROC) curve. The curve is computed by considering how the trade-off between sensitivity and false positive rate changes at a range of different cut-offs of class prediction probability $(M)$ returned by a given classifier. Here, the false positive rate is defined as follows:

$$
F P R=\frac{F P}{T N+F P}
$$

And, finally, given these definitions the area under (AUC) the ROC curve is described as follows:

$$
A U C(M)=\int_{\infty}^{-\infty} \operatorname{Sens}(M) \times\left(-F P R^{\prime}(M)\right) d M
$$

To verify that the performance of the classifier is robust, ROC-AUC measures were averaged across all cross-validation folds with the same fold number, and the resulting average curves, AUC values and their standard deviations are shown in Fig. 2. We also report the performance of the most recently released alternative method called pSumo-CD. The comparison was done by annotating all proteins in our dataset using the pSumo-CD web server. The resulting annotations were processed in an identical way to those of our method and the same set of four performance metrics were computed.

\section{Results and discussion}

The results of our evaluation are shown in Table 1, which showed that our method was able to outperform pSumo-CD on according to all metrics with exception of specificity. However, we note that there is a trade-off between the sensitivity and specificity measures, which can be realized by altering a threshold of the classifier at which a particular prediction is made. Notably, the sensitivity of pSUMO-CD is considerably lower, and more robust measures like accuracy (16.7\% higher) and Matthew's correlation coefficient ( 0.296 higher) are considerably better for our method. The difference between performances achieved for different $k$-folds validation schemes was also relatively small, indicating that over-fitting is likely to be relatively low. We also would like to point out that as pSumo-CD is, in essence, a machine learning method it will inherently have better performance on the samples from the original dataset used to train its model. Although from the description of the method we were able to deduce that there might be an overlap between our evaluation dataset and training dataset of pSumo-CD, not enough information was provided to allow us to identify and exclude these samples. Therefore, for this reason, the evaluation is likely to be biased in favor of pSumo-CD.

To compare the HseSUMO with pSumo-CD predictors, we have analyzed the sumoylation sites predicted by HseSUMO and pSumo-CD. (Additional file 1: Supplementary Table S1) shows the total number of sites predicted for 448 proteins analyzed in this paper. It is observed that out of 780 positives sites, HseSUMO correctly predicted 698 sites compared to 418 sites predicted by pSumo-CD. Thus, 35\% increase in the prediction ratio is noted for HseSUMO. Moreover, evaluating the performance of HseSUMO for different folds resulted with higher value of sensitivity for 

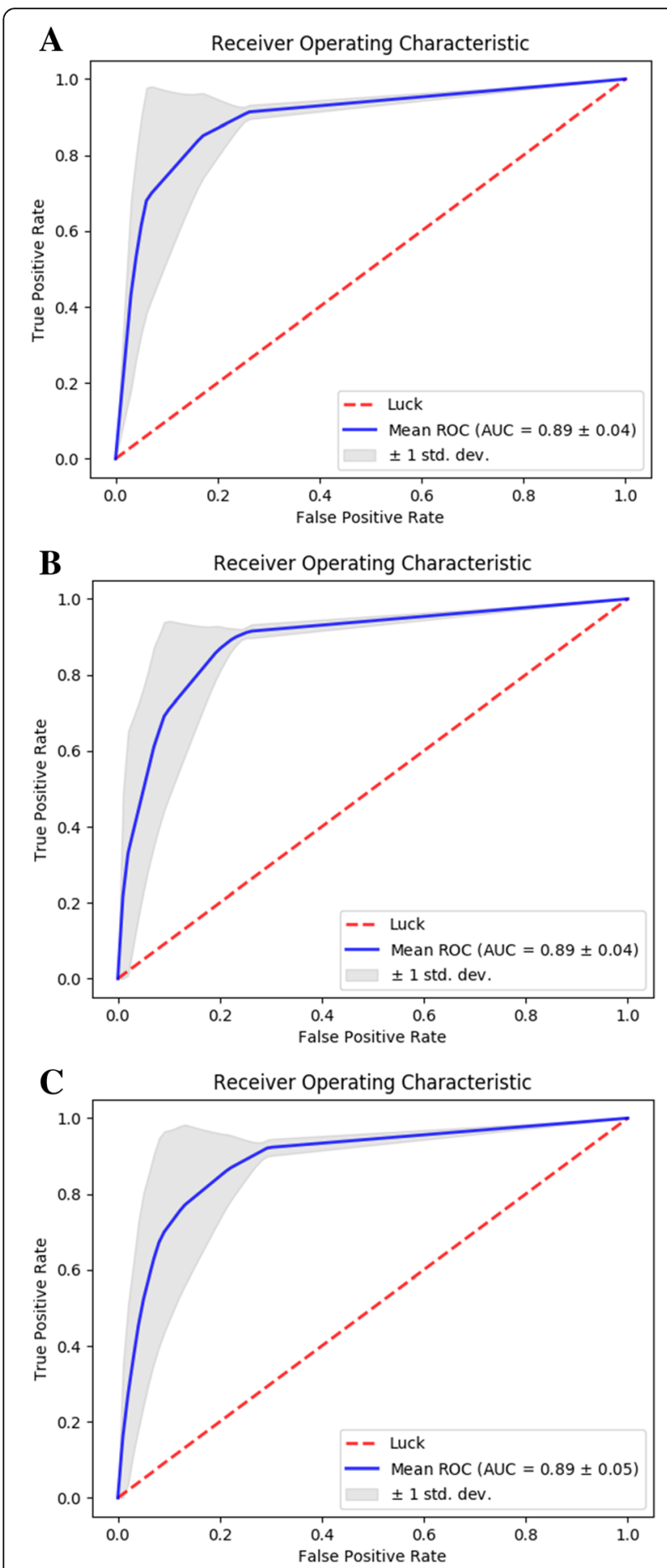

Fig. 2 Receiver operating characteristic curves of HseSUMO performance. Three panels show results for 6-fold (a), 8-fold (b), and 10-fold (c) evaluation schemes

10-fold cross-validation, while for 6-fold cross-validation a better performance was observed in terms of specificity, accuracy and MCC measures. Overall, there is a very little change in the performance measures for different folds when comparing HseSUMO and pSumo-CD.
Table 1 Performance evaluation of HseSUMO. CV refers to the cross-validation scheme for 6-fold, 8-fold and 10-fold

\begin{tabular}{lllll}
\hline Methods & Sensitivity & Specificity & Accuracy & MCC \\
\hline pSumo-CD & 0.536 & $\mathbf{0 . 9 2 1}$ & 0.728 & 0.494 \\
HseSUMO (CV- 6 & 0.895 & 0.895 & $\mathbf{0 . 8 9 5}$ & $\mathbf{0 . 7 9 0}$ \\
HseSUMO (CV- 8) & 0.897 & 0.879 & 0.888 & 0.777 \\
HseSUMO (CV- 10) & $\mathbf{0 . 9 0 4}$ & 0.872 & 0.888 & 0.776 \\
\hline
\end{tabular}

The highest values are depicted as bold faces

In detail, the highest sensitivity by HseSUMO was noted as 0.904 . The specificity was in the range of 0.872 and 0.895 . The specificity of pSumo-CD was the highest achieving at 0.921 , however, it provided sensitivity of 0.536 only. Thus, most of the sumoylation sites were not detected. The accuracy for HseSUMO was in the range of 0.888 and 0.895, however, for pSUMO-CD we achieved 0.728. The MCC measure was in the range 0.776 and 0.790 for HseSUMO, while pSUMO-CD was able to achieve 0.494 MCC score. In summary, we were able to achieve performance improvement of $36.5,16.7$, and $29.6 \%$ in terms of sensitivity, accuracy and MCC, respectively. However, pSumo-CD only showed high performance on specificity measure.

Although the metrics reported above are commonly used to evaluate the performance of particular machine learning classifiers, they are all subject to a limitation of only being applicable after a class prediction has been made. However, most classifiers can return a score rather than hard prediction and the final prediction is generated by applying a particular cut-off to that score. Depending on the cost attached to making an error of particular type different threshold can be chosen. Therefore, an analysis of the area under the ROC curve, which summarizes performance at all possible threshold can potentially be more useful. The results of the ROC-AUC analysis of our method are shown in Fig. 2; average AUC value for all fold numbers was recorded at 0.89 , indicating stable performance. In all cases, the higher standard deviation was associated with lower score cut-offs.

Our method was able to achieve highly competitive results despite forgoing pattern-based features that were key for many previous approaches, and, importantly, also performed well on an updated dataset that incorporated greater proportion of sites. These results indicate that structural features, e.g. HSE features are likely important for underlying biology of sumoylation mechanism and could be highly promising features for improvements in computational prediction of both sumoylation sites as well as other types of PTMs.

\section{Conclusions}

Despite recent progress in the development of better laboratory-based PTM detection methods, their experimental identification remains challenging. In this study, 
we propose a new, accurate method for prediction of sumoylation sites, an important type of PTM underlying multiple human diseases. Our method demonstrates the predictive power of features based on protein structure in case of sumoylation. This finding is of great interest, as all of the currently available methods in this area are based on features derived from some form of amino acid co-occurrence patterns, but recent experimental results indicate that over half of all known sumoylation sites are not associated with a clear amino acid motif [4]. Furthermore, to make current predictors more practical to the scientific community, a user friendly web-server is often developed [36, 64-66]. Therefore, we will make an effort to provide a flexible web-server for the method in the near future, which will undoubtedly contribute to enhance the ongoing work of experiment scientists and medical researchers alike. Meanwhile, interested researchers can access to the scripts and training matrices available at https://github.com/YosvanyLopez/HseSUMO.

\section{Additional file}

Additional file 1: Supplementary Table S1. Prediction of protein sequences by predictors (XLS $58 \mathrm{~kb}$ )

\section{Abbreviations}

ASA: Accessible surface area; AUC: Are under curve; CN: Contact number; FN: False negative; FP: False positive; HSE: Half-sphere exposure; HseSUMO: Sumoylation predictor using HSE of amino acids; MCC: Matthew's correlation coefficient; PTM: Post-translation modification; ROC: Receiver operating characteristics; TN: True negative; TP: True positive

\section{Acknowledgements}

Not applicable.

\section{Funding}

Publication costs were funded by the Grant-in-Aid for RIKEN IMS and CREST from the Japan Science and Technology Agency.

\section{Availability of data and materials}

The datasets used and analysed during the current study are publically available online at https://github.com/YosvanyLopez/HseSUMO

\section{Authors' contributions}

AIS and $A L$ conceived and wrote the first manuscript. $Y L$ and $A D$ performed analysis and experiments. HR, RS, AS and $\Pi$ contributed in manuscript writeup. All authors read and approved the final manuscript.

\section{Ethics approval and consent to participate}

Not applicable.

\section{Consent for publication}

Not applicable.

\section{Competing interests}

The authors declare that they do not have any competing interests.

\section{Publisher's Note}

Springer Nature remains neutral with regard to jurisdictional claims in published maps and institutional affiliations.

\section{Author details}

${ }^{1}$ Institute for Integrated and Intelligent Systems, Griffith University, Q Brisbane LD-4111, Australia. ' Laboratory for Medical Science Mathematics, RIKEN Center for Integrative Medical Sciences, Yokohama, Kanagawa, Japan. ${ }^{3}$ School of Engineering and Physics, Faculty of Science, Technology and Environment, University of the South Pacific, Suva, Fiji Islands. ${ }^{4}$ Genesis Institute of Genetic Research, Genesis Healthcare Co, Tokyo, Japan. ${ }^{5}$ Department of Computer Science, Morgan State University, Baltimore, MD, USA. ${ }^{6}$ School of Electrical and Electronics Engineering, Fiji National University, Suva, Fiji. ${ }^{7}$ Department of Medical Science Mathematics, Medical Research Institute, Tokyo Medical and Dental University, Tokyo, Japan. ${ }^{8}$ CREST, JST, Tokyo 113-8510, Japan.

Received: 25 May 2018 Accepted: 28 October 2018

Published: 18 April 2019

\section{References}

1. Mann M, Jensen ON. Proteomic analysis of post-translational modifications. Nat Biotechnol. 2003;21(3):255.

2. Jensen $\mathrm{ON}$. Interpreting the protein language using proteomics. Nat Rev Mol Cell Biol. 2006;7(6):391.

3. Warden SM, Richardson C, O'DONNELL J, Stapleton D, Witters LA. Posttranslational modifications of the $\beta-1$ subunit of AMP-activated protein kinase affect enzyme activity and cellular localization. Biochem J. 2001;354(2):275-83.

4. Hendriks IA, D'souza RC, Yang B, Verlaan-de Vries M, Mann M, Vertegaal AC. Uncovering global SUMOylation signaling networks in a site-specific manner. Nat Struct Mol Biol. 2014;21(10):927.

5. Pawson T, Scott JD. Protein phosphorylation in signaling-50 years and counting. Trends Biochem Sci. 2005;30(6):286-90.

6. Kessler BM, Edelmann MJ. PTMs in conversation: activity and function of deubiquitinating enzymes regulated via post-translational modifications. Cell Biochem Biophys. 2011;60(1-2):21-38.

7. Huber SC, Hardin SC. Numerous posttranslational modifications provide opportunities for the intricate regulation of metabolic enzymes at multiple levels. Curr Opin Plant Biol. 2004;7(3):318-22.

8. Swaney DL, Beltrao P, Starita L, Guo A, Rush J, Fields S, Krogan NJ, Villén J. Global analysis of phosphorylation and ubiquitylation cross-talk in protein degradation. Nat Methods. 2013;10(7):676.

9. Qing G, Lu Q, Xiong Y, Zhang L, Wang H, Li X, Liang X, Sun T. New opportunities and challenges of smart polymers in post-translational modification proteomics. Adv Mater. 2017;29(20). https://doi.org/10.1002/ adma.201604670.

10. Kamath KS, Vasavada MS, Srivastava S. Proteomic databases and tools to decipher post-translational modifications. J Proteome. 2011;75(1):127-44.

11. Flotho A, Melchior F. Sumoylation: a regulatory protein modification in health and disease. Annu Rev Biochem. 2013;82:357-85.

12. Steffan JS, Agrawal N, Pallos J, Rockabrand E, Trotman LC, Slepko N, Illes K, Lukacsovich T, Zhu Y-Z, Cattaneo E. SUMO modification of huntingtin and Huntington's disease pathology. Science. 2004;304(5667):100-4.

13. Krumova P, Weishaupt JH. Sumoylation in neurodegenerative diseases. Cell Mol Life Sci. 2013;70(12):2123-38.

14. Lee L, Sakurai M, Matsuzaki S, Arancio O, Fraser P. SUMO and Alzheimer's disease. NeuroMolecular Med. 2013;15(4):720-36.

15. Shuai K, Liu B. Regulation of JAK-STAT signalling in the immune system. Nat Rev Immunol. 2003:3(11):900.

16. Straus DS, Glass CK. Anti-inflammatory actions of PPAR ligands: new insights on cellular and molecular mechanisms. Trends Immunol. 2007;28(12):551-8.

17. Li M, Guo D, Isales CM, Eizirik DL, Atkinson M, She J-X, Wang C-Y. SUMO wrestling with type 1 diabetes. J Mol Med. 2005:83(7):504-13.

18. Kim Kl, Baek SH. SUMOylation code in cancer development and metastasis. Mol Cells. 2006;22(3):247-53.

19. Eifler K, Vertegaal AC. SUMOylation-mediated regulation of cell cycle progression and cancer. Trends Biochem Sci. 2015;40(12):779-93.

20. Rodriguez MS, Dargemont C, Hay RT. SUMO-1 conjugation in vivo requires both a consensus modification motif and nuclear targeting. J Biol Chem. 2001;276(16):12654-9.

21. Xue Y, Zhou F, Fu C, Xu Y, Yao X. SUMOsp: a web server for sumoylation site prediction. Nucleic Acids Res. 2006;34(suppl_2):W254-7.

22. Xu J, He Y, Qiang B, Yuan J, Peng X, Pan X-M. A novel method for high accuracy sumoylation site prediction from protein sequences. BMC Bioinformatics. 2008;9(1):8. 
23. Chen Y-Z, Chen Z, Gong Y-A, Ying G. SUMOhydro: a novel method for the prediction of sumoylation sites based on hydrophobic properties. PLoS One. 2012;7(6):e39195.

24. Zhao Q, Xie Y, Zheng Y, Jiang S, Liu W, Mu W, Liu Z, Zhao Y, Xue Y, Ren J. GPS-SUMO: a tool for the prediction of sumoylation sites and SUMOinteraction motifs. Nucleic Acids Res. 2014;42(W1):W325-30.

25. Yavuz AS, Sezerman OU. Predicting sumoylation sites using support vector machines based on various sequence features, conformational flexibility and disorder. BMC Genomics. 2014;15(9):S18

26. Jia J, Zhang L, Liu Z, Xiao X, Chou K-C. pSumo-CD: predicting sumoylation sites in proteins with covariance discriminant algorithm by incorporating sequencecoupled effects into general PseAAC. Bioinformatics. 2016;32(20):3133-41.

27. $X u$ Y, Ding $Y-X$, Deng N-Y, Liu L-M. Prediction of sumoylation sites in proteins using linear discriminant analysis. Gene. 2016;576(1):99-104.

28. Sharma R, Sharma A, Raicar G, Tsunoda T, Patil A. OPAL+: Length-Specific MoRF Prediction in Intrinsically Disordered Protein Sequences, Proteomics. 2018. https://doi.org/10.1002/pmic.201800058.

29. Saini H, Raicar G, Sharma A, Lal S, Dehzangi A, Lyons J, Paliwal KK, Imoto S, Miyano S. Probabilistic expression of spatially varied amino acid dimers into general form of Chous pseudo amino acid composition for protein fold recognition. J Theor Biol. 2015;380:291-8.

30. Sharma R, Dehzangi A, Lyons J, Paliwal K, Tsunoda T, Sharma A. Predict gram-positive and gram-negative subcellular localization via incorporating evolutionary information and physicochemical features into Chou's general PseAAC. IEEE Trans Nanobioscience. 2015;14(8):915-26.

31. Paliwal KK, Sharma A, Lyons J, Dehzangi A. Improving protein fold recognition using the amalgamation of evolutionary-based and structural based information. BMC Bioinformatics. 2014;15(Suppl 16):S12.

32. Lyons J, Dehzangi A, Heffernan R, Sharma A, Paliwal K, Sattar A, Zhou Y, Yang Y. Predicting backbone Calpha angles and dihedrals from protein sequences by stacked sparse auto-encoder deep neural network. J Comput Chem. 2014;35(28):2040-6.

33. Sharma A, Lyons J, Dehzangi A, Paliwal KK. A feature extraction technique using bi-gram probabilities of position specific scoring matrix for protein fold recognition. J Theor Biol. 2013;320:41-6.

34. Heffernan R, Dehzangi A, Lyons J, Paliwal K, Sharma A, Wang J, Sattar A, Zhou $Y$, Yang $Y$. Highly accurate sequence-based prediction of half-sphere exposures of amino acid residues in proteins. Bioinformatics. 2015;32(6):843-9.

35. Yang Y, Heffernan R, Paliwal K, Lyons J, Dehzangi A, Sharma A, Wang J, Sattar A, Zhou Y. SPIDER2: a package to predict secondary structure, accessible surface area and main-chain torsional angles by deep neural networks. Methods Mol Biol. 2017;1484:55-63.

36. Sharma R, Raicar G, Tsunoda T, Patil A, Sharma A. OPAL: prediction of MoRF regions in intrinsically disordered protein sequences. Bioinformatics. 2018; 34(11):1850-8.

37. Liu Z, Wang Y, Gao T, Pan Z, Cheng H, Yang Q, Cheng Z, Guo A, Ren J, Xue Y. CPLM: a database of protein lysine modifications. Nucleic Acids Res. 2014; 42(D1):D531-6.

38. Li W, Godzik A. Cd-hit: a fast program for clustering and comparing large sets of protein or nucleotide sequences. Bioinformatics. 2006; 22(13):1658-9.

39. Yen SJ, Lee YS. Under-sampling approaches for improving prediction of the minority class in an imbalanced dataset. Lect Notes Contr Inf. 2006:344:731-40.

40. Chakravarty S, Varadarajan R. Residue depth: a novel parameter for the analysis of protein structure and stability. Struct Fold Des. 1999;7(7):723-32.

41. Pollastri G, Baldi P, Fariselli P, Casadio R. Prediction of coordination number and relative solvent accessibility in proteins. Proteins. 2002:47(2):142-53.

42. Hamelryck T. An amino acid has two sides: a new $2 \mathrm{D}$ measure provides a different view of solvent exposure. Proteins. 2005;59(1):38-48.

43. Taherzadeh G, Zhou YQ, Liew AWC, Yang YD. Sequence-based prediction of protein-carbohydrate binding sites using support vector machines. J Chem Inf Model. 2016;56(10):2115-22.

44. Taherzadeh G, Yang YD, Zhang T, Liew AWC, Zhou YQ. Sequence-based prediction of protein-peptide binding sites using support vector machine. J Comput Chem. 2016;37(13):1223-9.

45. Jia JH, Liu Z, Xiao X, Liu BX, Chou KC. iSuc-PseOpt: identifying lysine succinylation sites in proteins by incorporating sequence-coupling effects into pseudo components and optimizing imbalanced training dataset. Anal Biochem. 2016a;497:48-56.

46. Liu Z, Xiao X, Qiu WR, Chou KC. iDNA-methyl: identifying DNA methylation sites via pseudo trinucleotide composition. Anal Biochem. 2015;474:69-77.
47. Chen w f p, ding $h \mid h$, Chou KC. iDNA6mA-PseKNC: identifying DNA N6methyladenosine sites using pseudo nucleotide composition. Anal Biochem. 2015;490:26-33.

48. Lopez Y, Sharma A, Dehzangi A, Lal SP, Taherzadeh G, Sattar A, Tsunoda T. Success: evolutionary and structural properties of amino acids prove effective for succinylation site prediction. BMC Genomics. 2018;19:923.

49. Dehzangi A, Lopez Y, Lal S, Taherzadeh G, Sattar A, Tsunoda T, Sharma A. Improving succinylation prediction accuracy by incorporating the secondary structure via helix, strand and coil, and evolutionary information from profile bigrams. PLoS One. 2018;13(2):e0191900.

50. Dehzangi A, Lopez Y, Lal SP, Taherzadeh G, Michaelson J, Sattar A, Tsunoda T, Sharma A. PSSM-Suc: accurately predicting succinylation using position specific scoring matrix into bigram for feature extraction. J Theor Biol. 2017:425:97-102.

51. Lopez Y, Dehzangi A, Lal SP, Taherzadeh G, Michaelson J, Sattar A, Tsunoda T. Sharma A. SucStruct: prediction of succinylated lysine residues by using structural properties of amino acids. Anal Biochem. 2017;527:24-32.

52. Sharma A, Imoto S, Miyano S. A top-r feature selection algorithm for microarray gene expression data. IEEE/ACM Trans Comput Biol Bioinform. 2012;9(3):754-64

53. Sharma A, Imoto S, Miyano S. A filter based feature selection algorithm using null space of covariance matrix for DNA microarray gene expression data. Curr Bioinforma. 2012;7(3):289-94.

54. Sharma A, Imoto S, Miyano S, Sharma V. Null space based feature selection method for gene expression data. Int J Mach Learn Cyb. 2012;3(4):269-76.

55. Chou KC. Some remarks on protein attribute prediction and pseudo amino acid composition (50th anniversary year review). J Theor Biol. 2011;273:236-47.

56. Chen W, Ding H, Feng P, Lin H, Chou KC. iACP: a sequence-based tool for identifying anticancer peptides. Oncotarget. 2016;7(13):16895-909.

57. Chen W, Feng P, Ding H, Lin H, Chou KC. iRNA-methyl: identifying N (6)methyladenosine sites using pseudo nucleotide composition. Anal Biochem. 2015;490:26-33.

58. Chen W, Feng P, Yang H, Ding H, Lin H, Chou K-C. iRNA-3typeA: identifying three types of modification at RNA's adenosine sites. Molecular Therapy Nucleic Acids. 2018:11:468-74.

59. Chen W, Feng P-M, Lin H, Chou K-C. iSS-PseDNC: identifying splicing sites using Pseudo dinucleotide composition. Biomed Res Int. 2014;2014:12

60. Sharma R, Dehzangi A, Lyons J, Paliwal K, Tsunoda T, Sharma A. Predict gram-positive and gram-negative subcellular localization via incorporating evolutionary information and physicochemical features into Chou's general PseAAC. IEEE transactions on nanobioscience. 2015;14(8):915-26.

61. Lyons J, Paliwal KK, Dehzangi A, Hefferman R, Tatsuhiko T, Sharma A. Protein fold recognition using HMM-HMM alignment and dynamic programming. J Theor Biol. 2016:393:67-74.

62. Dehzangi A, Hefterman R, Sharma A, Lyons J, Paliwal KK, Sattar A. Gram-positive and gram-negative protein subcellular localization by incorporating evolutionarybased descriptors into Chou's general PseAAC. J. Theor. Biol. 2015;364:284-94.

63. Chou KC. An unprecedented revolution in medicinal chemistry driven by the Progress of biological science. Curr Top Med Chem. 2017;17(21):2337-58.

64. Chen W, Feng PM, Deng EZ, Lin H, Chou KC. iTIS-PseTNC: a sequence-based predictor for identifying translation initiation site in human genes using pseudo trinucleotide composition. Anal Biochem. 2014:462:76-83.

65. Feng $P$, Ding $H$, Yang $H$, Chen W, Lin H, Chou KC. iRNA-PseColl: identifying the occurrence sites of different RNA modifications by incorporating collective effects of nucleotides into PseKNC. Mol Ther Nucleic Acids. 2017;7:155-63.

66. Feng $P$, Yang $H$, Ding $H$, Lin $H$, Chen W, Chou KC. iDNA6mA-PseKNC: identifying DNA N (6)-methyladenosine sites by incorporating nucleotide physicochemical properties into PseKNC. Genomics. 2018. https://doi.org/10. 1016/j.ygeno.2018.01.005.

Ready to submit your research? Choose BMC and benefit from:

- fast, convenient online submission

- thorough peer review by experienced researchers in your field

- rapid publication on acceptance

- support for research data, including large and complex data types

- gold Open Access which fosters wider collaboration and increased citations

- maximum visibility for your research: over $100 \mathrm{M}$ website views per year

At BMC, research is always in progress.

Learn more biomedcentral.com/submission 